\title{
The Wold Isomorphism for Cyclostationary Sequences
}

\author{
by \\ Harry Hurd \\ Timo Koski \\ Department of Statistics \\ Department of Mathematics \\ University of North Carolina \\ University of Linköping \\ Chapel Hill, N.C. 27599 -3260, USA \\ hurd@stat.unc.edu \\ S- 58183 Linköping SWEDEN \\ tikos@mai.liu.se
}

March 3, 2003

\begin{abstract}
In $1948 \mathrm{H}$. Wold introduced an isometric isomorphism between a Hilbert (linear) space formed from the weighted shifts of a numerical sequence and a suitable Hilbert space of values of a second order stochastic sequence. Motivated by a recent resurrection of the idea in the context of cyclostationary sequences and processes, we present the details of the Wold isomorphism between cyclostationary stochastic sequences and cyclostationary numerical sequences. We show how Hilbert-space representations of cyclostationary sequences are interpreted in the case of numerical CS sequences.
\end{abstract}

Key words: Periodically Correlated Time Series, Marcinkiewicz Spaces, Ergodic Properties, Linear Spaces Generated by Shifts of a Sequence. 


\section{Introduction}

In [34] Herman Wold identified an isometric isomorphism, to be called the Wold Isomorphism, between the Hilbert space of a stationary stochastic sequence and a Hilbert space formed from a numerical sequence. A numerical sequence is sometimes called a functional sequence or a time-series and may be envisioned as a doubly infinite sequence of numerical observations of some variable quantity made at equally spaced intervals of time. Wold was evidently motivated to show that methodologies involving linear processing of stationary stochastic sequences (such as linear prediction, innovations or spectral representations) would provide via this isomorphism the corresponding parallel results for a single time-series.

The notions of spectral analysis and linear prediction for a numerical sequence are connected to Norbert Wiener's theory of generalized harmonic analysis, see [30] or the reviews in [22] or [24]. In an early work [29], Wiener denoted numerical sequences as arrays, and the present authors give a more complete treatment of the spectral theory for the cyclostationary case in [18].

Lately the sphere of problems raised by Wold and Wiener has been resurrected by Gardner, see e.g. [6, pp. 179-181],[7, p. 377], [8], and [9] in the context of cyclostationary stochastic processes and cyclostationary numerical functions. Gardner introduced [6, pp. 347], in the spirit of Wold, the construction of an ensemble of random variables, with time index $t$ and ensemble index $s$ (shift) by

$$
X(t, s)=x_{t+s}
$$

where $\left\{x_{t}\right\}_{t=-\infty}^{\infty}$ is a numerical sequence of complex numbers. If the first and second moments of $X(t)$ are calculated using the fraction of time distributions, then these moments equal those determined by time averages of $\left\{x_{t}\right\}$, assuming the time averages exist.

The goal of this work was to see if a mapping in the precise sense of a standard Hilbert space isomorphism used by Wold [34] for the stationary case exists in the cyclostationary case. In presenting the affirmative result we have to examine carefully the domain and range spaces involved and the shift operators that shift the sequences by one period.

\subsection{Summary}

In section 2 we review and clarify the results of Wold [34] for stationary sequences. Here we are concerned with the space of two-sided complex valued sequences obtained by linear combinations of shifts of a given complex valued sequence $\mathbf{x}$. This linear space can be closed in a way to produce $H_{1}(\mathbf{x})$, a Hilbert subspace of a Marcinkiewicz space (see Bass [1]). This gives a little more insight into the nature of the sequence space that is isomorphic to the linear space of some second order stochastic sequence. Finally, it is important to note that the one place shift of a stationary sequence 
defines a unitary operator in the aforementioned Hilbert space of sequences, and this corresponds through the isomorphism to the unitary operator on the linear space of a stochastic sequence.

In section 3 we define CS (cyclostationary) numerical sequences in terms of the $N$ step mean and the $N$-step autocorrelation formed by averaging $N$-step subsequences. Using the $N$-step autocorrelation, a scalar product on the linear combinations of shifts of a given CS sequence $\mathbf{x}$ is defined and its closure $H_{N}(\mathbf{x})$ is the sequence space that is isomorphic to the linear space of some stochastic cyclostationary sequence. The result of Bass [1] again proves this sequence space to be a Hilbert sub-space of a Marcinkiewicz space of sequences. In this case, the one place shift of a CS sequence is no longer unitary in $H_{N}(\mathbf{x})$ but the $N$ place shift is unitary and corresponds through the isomorphism to the unitary operator of a stochastic cyclostationary sequence. We then apply the representations for stochastic CS sequences to the case of CS numerical sequences.

\subsection{Other related work}

Brillinger [3] gives a nice exposition of most of the issues about stationary functional sequences that are of concern to us. Bass [1] deals with "pseudo-random" functions (in continuous time) and gives a method for understanding the completion of a preHilbert space generated by a single numerical function.

Independently of Wold, and using a completely different technique, the problem of determining a stochastic process and its probability distributions, from a single numerical sequence were treated by Furstenberg [5] (see also Masani's review [23]). These ideas exceed our current framework as we shall only be concerned here with the $L_{2}$ theory. There are also related ideas in the papers $[31,32,33]$ and in $[21, \mathrm{p}$. 31, pp. $34-35]$.

Gardner incorporates in [10] the Wold isomorphism idea in a bigger discussion of basic scientific views about inference. He frames an approach to estimation theory that deals with a single sequence of numbers, the one we in fact use to form an estimate, and not with a set of sample paths, which is the view invoked by probabilistic models of inference. Gardner shows how these two views of inference effect the estimation of spectral density, which is a non-linear operation and thus neither covered by the Wold theory nor by this paper. The ideas in [10] have been extended to almost periodic and higher order cases [11, 12, 19, 20].

\subsection{Remarks}

In this presentation, one may interpret a stochastic sequence to be a general Hilbertspace valued sequence. We will use the stochastic terminology because under sufficient conditions of ergodicity, (this is the idea of Wold) almost all sequences of a stochastic CS sequence will be a functional CS sequence of the type required; we will men- 
tion some specific conditions in the conclusion. But our analysis does not require CS numerical sequences to be sample paths of stochastic CS sequences; the ergodic hypothesis merely allows the construction of examples.

\section{The Stationary Case}

\subsection{Preliminaries}

Let $\mathbf{x}=\left\{x_{j}\right\}_{j=-\infty}^{\infty}$ designate a numerical sequence of complex numbers. If $\Pi_{j}\{\cdot\}$ is the coordinate map i.e.

$$
\Pi_{j}\{\mathbf{x}\}:=x_{j}
$$

and $\lambda$ is a complex number, then the operations of addition and multiplication by a scalar are defined in terms of the coordinate maps as

$$
\begin{aligned}
\Pi_{j}\{\mathbf{x}+\mathbf{y}\} & =x_{j}+y_{j}, \\
\Pi_{j}\{\lambda \cdot \mathbf{x}\} & =\lambda x_{j} .
\end{aligned}
$$

We define also the mean

$$
\mu(\mathbf{x}):=\lim _{n \rightarrow \infty} \frac{1}{2 n+1} \sum_{j=-n}^{n} x_{j}
$$

and the norm

$$
\|\mathbf{x}\|_{1}^{2}:=\lim _{n \rightarrow \infty} \frac{1}{2 n+1} \sum_{j=-n}^{n}\left|x_{j}\right|^{2}
$$

whenever the limits exist. Similarly we introduce for a pair of numerical sequences $\mathbf{x}$ and $\mathbf{y}$ the scalar product

$$
(\mathbf{x}, \mathbf{y})_{1}:=\lim _{n \rightarrow \infty} \frac{1}{2 n+1} \sum_{j=-n}^{n} x_{j} \bar{y}_{j},
$$

as soon as this exists, where $\bar{y}_{i}$ denotes the complex conjugation of an element in the sequence $\mathbf{y}$. Whenever the limits (2.1), (2.2), (2.3) exist, then taking (2.3) to be specific, then for any integer $q$, the limit

$$
\lim _{n \rightarrow \infty} \frac{1}{2 n+1} \sum_{j=-n}^{n} x_{j+q} \bar{y}_{j+q}
$$

exists and is equal to (2.3); that is, the limits are independent of the origin. Using Cauchy's inequality for complex sequences we have $\left|(\mathbf{x}, \mathbf{y})_{1}\right| \leq\|\mathbf{x}\|_{1}^{2} \cdot\|\mathbf{y}\|_{1}^{2}$ when all the necessary limits exist. 
The autocorrelation of $\mathbf{x}$ is the sequence $\left\{r_{k}\right\}_{k=-\infty}^{\infty}$ where

$$
r_{k}:=r_{k}(\mathbf{x}):=\lim _{n \rightarrow \infty} \frac{1}{2 n+1} \sum_{j=-n}^{n} x_{j+q} \bar{x}_{j+q+k},
$$

if the limit exists, and by our previous comment it is independent of the integer $q$. At any value of $k$ for which $r_{k}$ exists, $r_{-k}=\bar{r}_{k}$ and $\left|r_{k}\right| \leq r_{0}=(\mathbf{x}, \mathbf{x})_{1}<\infty$.

We denote $\mathcal{S}$ as the forward (left) shift operator, defined in the set of doubly infinite numerical sequences by the action

$$
\Pi_{j}\{\mathcal{S} \mathbf{x}\}=x_{j+1}
$$

for every $j$. The inverse $\mathcal{S}^{-1}$ exists and is simply given as the backward (right) shift, since the sequences are two-sided. The operation of $j$ consecutive shifts to the left is defined inductively as the $j^{\prime}$ th power of $\mathcal{S}, \mathcal{S}^{j}$ as is $\mathcal{S}^{-j}$. Hence we may write

$$
r_{k}(\mathbf{x})=\left(\mathbf{x}, \mathcal{S}^{k} \mathbf{x}\right)_{1} .
$$

\subsection{Stationary numerical sequences}

We define next (c.f. [33, p. 98]) the notion of a stationary numerical sequence in Wold's sense.

Definition 2.1 A sequence $\mathbf{x}=\left\{x_{j}\right\}_{j=-\infty}^{j=\infty}$ such that

1. the mean $\mu(\mathbf{x})$ exists as a finite number,

2. the autocorrelation $r_{k}(\mathbf{x})$ exists for every integer $k$,

is called a stationary numerical sequence.

Example 2.1 The numerical sequence $\mathbf{e}_{\alpha}=\left\{e^{i \alpha j}\right\}_{j=-\infty}^{\infty}(i=\sqrt{-1})$ is stationary for any real $\alpha$. Two sequences $\mathbf{e}_{\alpha}$ and $\mathbf{e}_{\beta}$ are orthonormal with respect to $(\cdot, \cdot)_{1}$ for $\alpha \neq \beta$.

Before completing our discussion of the Wold isomorphism we will first consider jointly stationary numerical sequences.

First, if $\left\{\mathbf{z}^{(k)}, k=0,1, \ldots, K-1\right\}$ is a collection of numerical sequences, we define

$$
\underline{\mathbf{z}}:=\left(\begin{array}{c}
\mathbf{z}^{(0)} \\
\mathbf{z}^{(1)} \\
\vdots \\
\mathbf{z}^{(K-1)}
\end{array}\right) .
$$


Definition 2.2 A numerical vector sequence $\underline{\mathbf{z}}$, with $K$ components $\mathbf{z}^{(k)}=\left\{z_{j}^{(k)}\right\}_{j=-\infty}^{\infty} k=$ $0,1, \ldots, K-1$, is stationary if the component sequences are jointly stationary in the sense that the cross correlations defined by

$$
\phi_{r, s}(p-q):=\lim _{n \rightarrow \infty} \frac{1}{2 n+1} \sum_{j=-n}^{n} z_{j+p}^{(r)} \bar{z}_{j+q}^{(s)},
$$

exist for $0 \leq r, s \leq K-1$ and all $p, q \in \mathbf{Z}$.

We note here that when the limit exists for some $p, q$, it will depend only on the difference $p-q$. Also, the collection $\left\{\phi_{r, s}(k), 0 \leq r, s \leq K-1\right\}$ is a crosscorrelation matrix: for any $n$, collections $\left\{\alpha_{1}, \alpha_{2}, \ldots, \alpha_{n} \in \mathbf{C}\right\},\left\{\tau_{1}, \tau_{2}, \ldots, \tau_{n} \in \mathbf{Z}\right\}$ and $\left\{k_{1}, k_{2}, \ldots, k_{n} \in[0,1, \ldots, K-1]\right\}$, we have

$$
\sum_{p=1}^{n} \sum_{q=1}^{n} \alpha_{p} \overline{\alpha_{q}} \phi_{k_{p}, k_{q}}\left(\tau_{p}-\tau_{q}\right) \geq 0 .
$$

In the case of stationary stochastic sequences $\left\{\xi_{n}, n \in \mathbf{Z}\right\}$, the sequences $\xi^{(k)}=$ $\left\{\xi_{n N+k}, n \in \mathbf{Z}, k=0,1, \ldots, N-1\right\}$ formed by sampling $\left\{\xi_{n}, n \in \mathbf{Z}\right\}$ at intervals of length $N$ are jointly stationary sequences, and this occurs for every $N$. In the case of numerical sequences, it does not automatically occur that subsequences formed by periodic sampling are stationary because the needed limits may not exist. As an example, let $\left\{x_{n}\right\}$ be a real sequence taking the values $-1,1$ whose mean does not converge. Then construct $\left\{y_{n}\right\}$ by $\ldots, x_{-1},-x_{-1}, x_{0},-x_{0}, x_{1},-x_{1}, \ldots$. We can see $\mu(\mathbf{y})$ exists but the limit defining $\mu\left(\left\{y_{2 j}\right\}\right)=\mu(\mathbf{x})$ does not exist. Thus we are led to the following.

Definition 2.3 A sequence $\mathbf{x}=\left\{x_{j}\right\}_{j=-\infty}^{\infty}$ is called totally stationary if the sequences $\left\{\mathbf{x}^{(k)}, k=0,1, \ldots, N-1\right\}$ given by

$$
\Pi_{j}\left\{\mathbf{x}^{(k)}\right\}=\Pi_{k+j N}\{\mathbf{x}\}
$$

are jointly stationary in the sense expressed in (2.9) for positive $N$.

A totally stationary sequence is 1-stationary, that is, stationary in the sense of Definition 2.1.

\subsection{The Wold Isomorphism}

In order to introduce the Wold isomorphism let us now assume that we have a stationary numerical sequence $\mathbf{x}$. Since the autocorrelation exists, then for any scalar $\lambda$,

$$
\left\|\mathbf{x}+\lambda \mathcal{S}^{k} \mathbf{x}\right\|_{1}
$$


exists and is finite. Thus we may introduce the linear space $M_{1}(\mathbf{x})$ defined as the (finite) linear span

$$
\begin{aligned}
M_{1}(\mathbf{x}) & =\operatorname{sp}\left\{\mathcal{S}^{k} \mathbf{x} \mid k \text { integer }\right\} \\
& =\left\{\mathbf{y}=\sum_{j=-p}^{p} \lambda_{j} \mathcal{S}^{j} \mathbf{x}, \text { arbitrary } p, \lambda_{j}\right\},
\end{aligned}
$$

and on this linear space $\left(\mathbf{x}_{1}, \mathbf{x}_{2}\right)_{1}$ of (2.3) is seen to have the properties of symmetry, additivity and homogeneity; that is, (a) $\left(\mathbf{x}_{1}, \mathbf{x}_{2}\right)_{1}=\overline{\left(\mathbf{x}_{2}, \mathbf{x}_{1}\right)_{1}}$, (b) $\left(\mathbf{x}_{1}+\mathbf{x}_{2}, \mathbf{x}_{3}\right)_{1}=$ $\left(\mathbf{x}_{1}, \mathbf{x}_{2}\right)_{1}+\left(\mathbf{x}_{2}, \mathbf{x}_{3}\right)_{1}$ and (c) $\left(\alpha \mathbf{x}_{1}, \mathbf{x}_{2}\right)_{1}=\alpha\left(\mathbf{x}_{1}, \mathbf{x}_{2}\right)_{1}$ for every complex $\alpha$ and all $\mathbf{x}_{1}, \mathbf{x}_{2}, \mathbf{x}_{3}$ in $M_{1}(\mathbf{x})$. In order for $\left(\mathbf{x}_{1}, \mathbf{x}_{2}\right)_{1}$ to be positive definite in the sense that $\|\mathbf{x}\|_{1}^{2}=0 \Longleftrightarrow \mathbf{x}=0$ we interpret $\mathbf{0}$ as the (equivalence) class of sequences that are equivalent to the zero sequence (the complex sequence with $x_{j}=0$ for every $j$ ). This expresses the sense of uniqueness obtained by the scalar product (2.3). For example any two sequences $\mathbf{x}_{1}, \mathbf{x}_{2}$ for which $\mathbf{z}=\mathbf{x}_{1}-\mathbf{x}_{2} \in \ell_{2}$ are clearly equivalent because $\mathbf{z}$ satisfies $\left\|\mathbf{x}_{1}-\mathbf{x}_{2}\right\|_{1}=0$. Thus any finite sequence is equivalent to $\mathbf{0}$ and any two sequences differing in a finite number of positions are equivalent.

In the sequel $\mathbf{x}$ will denote (the representative of ) any equivalence class in $M_{1}(\mathbf{x})$. By the definitions of the linear operations and the inner product $(\cdot, \cdot)_{1}$ we have thus established $M_{1}(\mathbf{x})$ as a pre-Hilbert space.

Let us note that the autocorrelation $\left\{r_{k}(\mathbf{x})\right\}_{k=-\infty}^{\infty}$ is a non-negative definite sequence and is identifiable as the autocorrelation function of some (weakly) stationary stochastic sequence $\xi=\left\{\xi_{j}\right\}_{j=-\infty}^{\infty}$ defined on some probability space $(\Omega, \mathcal{F}, P)$. From the sequence $\xi$ we can determine a Hilbert space $H(\xi)$, the linear span of $\xi_{t}$ closed with respect to mean square norm $\|\eta\|_{H(\xi)}=\sqrt{E|\eta|^{2}}$, where $E$ is expectation with respect to the probability measure $P$.

Let us consider a linear map $J$ defined on $M_{1}(\mathbf{x})$ and assuming values in the linear $\operatorname{span} \operatorname{sp}\left\{\xi_{k}, k \in \mathbf{Z}\right\}$ of the sequence $\xi$ by

$$
J\left(\lambda \mathcal{S}^{p} \mathbf{x}+\mu \mathcal{S}^{k} \mathbf{x}\right)=\lambda \xi_{p}+\mu \xi_{k}
$$

for arbitrary complex $\lambda, \mu$ and integers $k, p$. This defines the Wold isomorphism $^{1}$ between the pre-Hilbert space $M_{1}(\mathbf{x})$ and it's image $J M_{1}(\mathbf{x})=\operatorname{sp}\left\{\xi_{k}, k \in \mathbf{Z}\right\}$, which is dense in $H(\xi)$. If we denote $H_{1}(\mathbf{x})$ as an abstract completion of $M_{1}(\mathbf{x})$ with respect to $\|\cdot\|_{1}$, then by the continuity (boundedness) of $J$ on $M_{1}(\mathbf{x}), J$ extends to a map from $H_{1}(\mathbf{x})$ to $H(\xi)$ and is the desired Hilbert space isomorphism. The elements of $H(\xi)$ are all $L_{2}(\Omega, \mathcal{F}, P)$ random variables, but there arises a question about the interpretation of the limit points of $M_{1}(\mathbf{x})$. That is, are we left only to define the closure as an abstract completion as in [26, p. 121, 124 - 125], or does there exist a more concrete interpretation?

\footnotetext{
${ }^{1}$ It is an isomorphism in the sense that it is a bijection (invertible) and preserves inner products, and hence is also linear [26].
} 
A positive answer is provided by J. Bass [1, pp.33- 35] for the case in which our sequences are replaced by functions in a Marcinkiewicz space $\mathcal{M}^{2}$. Adapting the result of Bass, it follows that the completion of the pre-Hilbert space $M_{1}(\mathbf{x})$ with respect to the norm

$$
\|\mathbf{x}\|_{\mathcal{M}^{2}}^{2}=\limsup _{n \rightarrow \infty} \frac{1}{2 n+1} \sum_{j=-n}^{n}\left|x_{j}\right|^{2}
$$

is in fact a Hilbert space with respect to the scalar product $(\mathbf{x}, \mathbf{y})_{1}$. That is, if $\mathbf{y} \in H_{1}(\mathbf{x})$ then $\mathbf{y} \in \mathcal{M}^{2}$ but also that if $\mathbf{y}$ and $\mathbf{z} \in H_{1}(\mathbf{x})$ then $(\mathbf{y}, \mathbf{z})_{1}$ exists as a limit.

Hence we have obtained the following assertion.

Proposition 2.2 Let $\mathrm{x}$ be a stationary numerical sequence having autocorrelation sequence $\left\{r_{k}(\mathbf{x})\right\}_{k=-\infty}^{\infty}$ and let $J$ be the map from $M_{1}(\mathbf{x})$ into $\operatorname{sp}\left\{\xi_{k}, k \in \mathbf{Z}\right\} \subset H(\xi)$ defined for arbitrary complex $\lambda, \mu$ and integers $k, p$ by (2.14). Then $J$ can be extended as an isometric Hilbert space isomorphism from a complex Hilbert space $H_{1}(\mathbf{x}) \supset$ $M_{1}(\mathbf{x})$ to the complex Hilbert space $H(\xi)$, the closed linear span of a wide sense stationary sequence $\xi$ that has autocorrelation function equal to $\left\{r_{k}(\mathbf{x})\right\}_{k=-\infty}^{\infty}$.

The assignment

$$
J \mathcal{S}^{k} \mathbf{x}=\xi_{k}, k \text { an integer }
$$

is conceptually utilized throughout Wold's paper [34], but is never explicitly stated nor is there any comment about how one is to think about the closure of $M_{1}(\mathbf{x})$.

Let us recall that in $H(\xi)$ there exists a unitary operator $U$ such that

$$
U \xi_{j}=\xi_{j+1}
$$

(see e.g. [27, p. 14]). Hence the Wold isomorphism $J$ leads to

$$
J \mathcal{S}^{k} \mathbf{x}=\xi_{k}=U^{k} \xi_{0}=U^{k} J \mathbf{x}
$$

which justifies the expression

$$
U=J \mathcal{S} J^{-1} \text {. }
$$

Thus in the stationary case $U$ and $\mathcal{S}$ are unitarily equivalent or similar operators (see [28, p. 242] and [26, p. 193]).

It is natural to say that two $L_{2}$ stochastic sequences $\left\{\xi_{j}\right\}$ and $\left\{\eta_{j}\right\}$ are equivalent if and only if the random variables are identical: $\left\|\xi_{j}-\eta_{j}\right\|_{L^{2}}=0$ for every $j$. In the case of numerical sequences, the relationship

$$
\left\|\mathcal{S}^{j} \mathbf{x}-\mathcal{S}^{j} \mathbf{y}\right\|_{1}=0
$$

is valid for every $j$ if it is valid for $j=0$. 


\subsection{The Spectral Representation of Stationary Numerical Se- quences}

Having established that $\mathcal{S}$ is a unitary operator on $H_{1}(\mathbf{x})$, we can use the spectral representation for stationary sequences (in any Hilbert space) to write

$$
\mathcal{S}^{k} \mathbf{x}=\int_{0}^{2 \pi} \exp (i \lambda k) d Z(\lambda)
$$

where $Z(\lambda)=E(\lambda) \mathbf{x}$ with $E(\lambda)$ denoting a spectral family of projection operators on $H_{1}(\mathbf{x})$. The interpretation of (2.21) is that the quantity

$$
\left\|\mathcal{S}^{k} \mathbf{x}-\sum_{m=1}^{n} \exp \left(i \lambda_{m} k\right)\left[Z\left(\lambda_{m}\right)-Z\left(\lambda_{m-1}\right)\right]\right\|_{1}^{2}
$$

converges to 0 as the $n \rightarrow \infty$ and norm of the partition $\left\{0=\lambda_{0}, \lambda_{1}, \ldots, \lambda_{n}=2 \pi\right\}$ tends to zero.

The sense of the approximation given in (2.22) (see Brillinger [3, Theorem 3.9.1]) is that the increments $\left[Z\left(\lambda_{m}\right)-Z\left(\lambda_{m-1}\right)\right]=\left[E\left(\lambda_{m}\right)-E\left(\lambda_{m-1}\right)\right] \mathbf{x}$ are elements of $H_{1}(\mathbf{x})$ and thus are sequences themselves. Hence $\exp \left(i \lambda_{m} k\right)\left[Z\left(\lambda_{m}\right)-Z\left(\lambda_{m-1}\right)\right]$ is the increment sequence $Z\left(\lambda_{m}\right)-Z\left(\lambda_{m-1}\right)$ with every element multiplied by the scalar $\exp \left(i \lambda_{m} k\right)$. It is important to observe that the parameter $k$ is no longer associated with time, as in the stochastic case, but with the number of positions the sequence $\mathbf{x}$ is shifted.

\section{The Cyclostationary Case}

\subsection{Cyclostationary Numerical Sequences}

Here we will give the definition of cyclostationary numerical sequences. A key idea in the definition of numerical cyclostationary sequences (see [6]), is the use of an $N$-step mean and an $N$-step autocorrelation.

Definition 3.1 The $N$-step mean of a numerical sequence $\mathbf{x}$ with starting point $m$ is the quantity

$$
\mu_{m}^{(N)}(\mathbf{x}):=\lim _{M \rightarrow \infty} \frac{1}{2 M+1} \sum_{j=-M}^{M} x_{j N+m}
$$

when the limit exists.

It follows from the independence of the origin that if $\mu_{m}^{(N)}(\mathbf{x})$ exists, then $\mu_{m+k N}^{(N)}(\mathbf{x})$ also exists for every integer $k$ and

$$
\mu_{m}^{(N)}(\mathbf{x})=\mu_{m+k N}^{(N)}(\mathbf{x})
$$

for all $m, n$. So if the $N$-step mean $\mu_{m}^{(N)}(\mathbf{x})$ exists for $m=0,1, \ldots, N-1$, it exists for all $m$ and (3.2) is true for all $m$. 
Definition 3.2 The $N$-step autocorrelation of a numerical sequence $\mathbf{x}$ is the quantity obtained from the $N$-step scalar product between the numerical sequences $\mathcal{S}^{m} \mathbf{x}$ and $\mathcal{S}^{n} \mathbf{x}$ :

$$
R_{m, n}^{(N)}(\mathbf{x}):=\lim _{M \rightarrow \infty} \frac{1}{2 M+1} \sum_{j=-M}^{M} x_{j N+m} \bar{x}_{j N+n}
$$

when the limit exists.

Again it follows from the independence of the origin that if $R_{m, n}^{(N)}(\mathbf{x})$ exists, then $R_{m+k N, n+k N}^{(N)}(\mathbf{x})$ exists for every integer $k$ and

$$
R_{m, n}^{(N)}(\mathbf{x})=R_{m+k N, n+k N}^{(N)}(\mathbf{x}) .
$$

So if the $N$-autocorrelation $R_{m, n}^{(N)}(\mathbf{x})$ exists for $m=0,1, \ldots, N-1, n \in \mathbf{Z}$, it exists for all $m, n$ and (3.4) is true for all $m, n$.

Definition 3.3 A sequence $\mathbf{x}=\left\{x_{j}\right\}_{j=-\infty}^{\infty}$ such that

1. the $N$-step mean exists for every $m$ (and thus satisfies (3.2) for all $m$ );

2. for $m=0,1, \ldots, N-1, n \in \mathbf{Z}$, the autocorrelation kernel exists (and thus satisfies (3.4) for all $m, n$;

is defined to be cyclostationary with period $N$.

For the purpose of notation, we shall say that a cyclostationary sequence with period $N$ is $C S(N)$ and we note that $\mathrm{CS}(1)$ corresponds to stationarity in the sense of section 2.2 above.

Observe that the $N$-step mean and autocorrelation are formed by periodic sampling of $\mathbf{x}$. In fact this suggests that one may view a CS numerical sequence as a sampling of shifts of some sequence $\mathbf{x}$, rather than as $\mathbf{x}$ itself.

There is another condition on the autocorrelation that is often found in literature on cyclostationarity; we now make precise its equivalence to item 2 above.

Proposition 3.1 A necessary and sufficient condition for the existence of $R_{m, n}^{(N)}(\mathbf{x})$ for all $m, n$ is the existence of

$$
\left.B_{k, \tau}^{(N)}(\mathbf{x}):=\lim _{M \rightarrow \infty} \frac{1}{2 M+1} \sum_{j=-M}^{M} x_{j+\tau} \bar{x}_{j} \exp (-i 2 \pi k j / N)\right)
$$

for $k=0,1, \ldots, N-1, \tau \in \mathbf{Z}$. 
Proof: It is not difficult to show, using the invertibility of discrete Fourier transforms, that a collection of complex numbers $\left\{R_{m, n}^{(N)}\right\}, m, n \in \mathbf{Z}$ satisfy $R_{m, n}^{(N)}=R_{m+N, n+N}^{(N)}$ if and only if there exist a family of Fourier coefficient sequences $\left\{B_{k, \tau}^{(N)}, \tau \in \mathbf{Z}\right\}_{k=0}^{N-1}$ for which

$$
R_{m, n}^{(N)}=\sum_{k=0}^{N-1} B_{k, m-n}^{(N)} \exp (i 2 \pi k n / N) .
$$

The coefficient sequences are determined by

$$
B_{k, \tau}^{(N)}=\frac{1}{N} \sum_{n=0}^{N-1} R_{n+\tau, n}^{(N)} \exp (-i 2 \pi k n / N)
$$

The necessity of our claim follows from the fact that if the autocorrelation exists for all $m, n$, then

$$
\begin{aligned}
B_{k, \tau}^{(N)}(\mathbf{x}) & =\lim _{M \rightarrow \infty} \frac{1}{2 M+1} \sum_{j=-M}^{M} x_{j+\tau} \bar{x}_{j} \exp (-i 2 \pi k j / N) \\
& =\frac{1}{N} \sum_{n=0}^{N-1} \lim _{M^{\prime} \rightarrow \infty} \frac{1}{2 M^{\prime}+1} \sum_{j^{\prime}=-M^{\prime}}^{M^{\prime}} x_{j^{\prime} N+n+\tau} \bar{x}_{j^{\prime} N+n} \exp (-i 2 \pi k n / N) \\
& =\frac{1}{N} \sum_{n=0}^{N-1} R_{n+\tau, n}^{(N)}(\mathbf{x}) \exp (i 2 \pi k n / N)
\end{aligned}
$$

where we use the fact that every $j \in \mathbf{Z}$ in the first sum has a unique representation $j=j^{\prime} N+n(j)$ for $0 \leq n \leq N-1$ and

$$
\exp [-i 2 \pi k j / N]=\exp \left[-i 2 \pi k\left(j^{\prime} N+n(j)\right) / N\right]=\exp [-i 2 \pi k n(j) / N] .
$$

The sufficiency may be deduced in the same manner by starting with the existence of the $B_{k, \tau}(\mathbf{x}), k=0,1, \ldots N-1$ and showing with the help of

$$
R_{m, n}^{(N)}(\mathbf{x})=\sum_{k=0}^{N-1} B_{k, m-n}^{(N)}(\mathbf{x}) \exp (i 2 \pi k n / N)
$$

that the averages $R_{m, n}^{(N)}(\mathbf{x})$ must exist.

Remark 3.1 The quantity $B_{k, \tau}^{(N)}(\mathbf{x})$ is identically zero for $k \neq 0$ if and only if $R_{m, n}^{(N)}(\mathbf{x})$ depends only on $m-n$, as is seen by (3.6) and (3.7). This condition defines a stationary subclass of $\operatorname{CS}(N)$. We refer to this subclass as the set of $N$-stationary numerical sequences. 


\subsection{Examples}

Example 3.2 The stationary numerical sequence $\mathbf{e}_{\alpha}$ given in Example 2.1 above is also $C S(N)$ for arbitrary positive $N$ and also totally stationary.

In the following examples we will often use the idea of a strictly periodic sequence. A sequence $\mathbf{f}=\left\{f_{j}\right\}_{j=-\infty}^{\infty}$ will be called strictly periodic with period $N$ if $f_{j}=f_{j+N}$ for all $j$, and this holds for no smaller value of $N$.

Example 3.3 A modulated exponential CS sequence. The numerical sequence $\mathbf{x}$ obtained using amplitude modulation of $\mathbf{e}_{\alpha}$ in Example 2.1 by a strictly periodic numerical sequence $\mathbf{f}$ and defined as

$$
x_{j}=f_{j} e^{i \alpha j}
$$

for any integer $j$, is $C S(N)$, as is easily seen.

Example 3.4 Amplitude scale modulation. Suppose $\mathrm{y}$ is a totally stationary numerical sequence. If $\mathbf{f}$ is strictly periodic with period $N$, then consider the product sequence

$$
x_{j}=f_{j} y_{j}
$$

for any integer $j$. Then for any $m, n$,

$$
\frac{1}{2 M+1} \sum_{j=-M}^{M} x_{j N+m} \bar{x}_{j N+n}=\frac{1}{2 M+1} \sum_{j=-M}^{M} f_{j N+m} y_{j N+m} \overline{f_{j N+n} y_{j N+n}}
$$

converges using the notation in (2.9) to

$$
R_{m, n}^{(N)}(\mathbf{x})=f_{m} \overline{f_{n}} \phi_{m-n}(0)=R_{m+N, n+N}^{(N)}(\mathbf{x}) .
$$

Taking $f_{j}=1$ for all $j$ shows that a totally stationary numerical sequence is $C S(N)$.

Example 3.5 Time scale modulation. Suppose y is a totally stationary sequence and $f: \mathbf{Z} \rightarrow \mathbf{Z}$ is strictly periodic with period $N$. Then the periodically rearranged sequence

$$
x_{j}=y_{\left(j+f_{j}\right)}
$$

is $\operatorname{CS}(N)$. Indeed, consider for any $m, n$

$$
\frac{1}{2 M+1} \sum_{j=-M}^{M} x_{j N+m} \bar{x}_{j N+n}=\frac{1}{2 M+1} \sum_{j=-M}^{M} y_{\left(j N+m+f_{j N+m}\right)} \overline{y_{\left(j N+n+f_{j N+n}\right)}}
$$

which converges in the notation of $(2.9)$ to

$$
R_{m, n}^{(N)}(\mathbf{x})=\phi_{m+f_{m}-n-f_{n}}(0)=R_{m+N, n+N}^{(N)}(\mathbf{x})
$$


Example 3.6 Periodic Mixtures of Jointly Stationary Sequences. This is a generalization of the amplitude modulation model. It is possible to show that all cyclostationary numerical sequences can be expressed in a similar form [18].

Suppose $\left\{\mathbf{y}^{k}, k=0,1, \ldots, K-1\right\}$ is a collection of jointly totally stationary sequences and $\left\{\mathbf{f}^{k}, k=0,1, \ldots, K-1\right\}$ is a collection of strictly periodic sequences all with period $N$. Then the sum (a sequence)

$$
x_{j}=\sum_{k=0}^{K-1} f_{j}^{k} y_{j}^{k}
$$

is $\mathrm{CS}(N)$. Indeed, consider for any $m, n$

$$
\frac{1}{2 M+1} \sum_{j=-M}^{M} x_{j N+m} \bar{x}_{j N+n}=\frac{1}{2 M+1} \sum_{j=-M}^{M} \sum_{k=1}^{K-1} \sum_{k^{\prime}=0}^{K-1} f_{j N+m}^{k} y_{j N+m}^{k} \overline{f_{j N+n}^{k^{\prime}} y_{j N+n}^{k^{\prime}}}
$$

which converges to

$$
R_{m, n}^{(N)}(\mathbf{x})=\sum_{k=0}^{K-1} \sum_{k^{\prime}=0}^{K-1} f_{m}^{k} \overline{f_{n}^{k^{\prime}}} \phi_{k, k^{\prime}}(m-n)=R_{m+N, n+N}^{(N)}(\mathbf{x}) .
$$

\subsection{Preliminary properties}

In this section we recapitulate some essential properties that the autocorrelation $R_{m, n}^{(N)}(\mathbf{x})$ possesses and show the close connection of CS numerical sequences, as in the case of random sequences [13], to stationary vector sequences.

Lemma $3.7(m, n) \mapsto R_{m, n}^{(N)}(\mathbf{x})$ is non-negative definite.

Proof: We need to show

$$
\sum_{p=0}^{k} \sum_{q=0}^{k} \lambda_{p} \bar{\lambda}_{q} R_{p, q}^{(N)}(\mathbf{x}) \geq 0
$$

for arbitrary integer $k$ and arbitrary complex numbers $\left\{\lambda_{p}\right\}_{p=0}^{k}$.

But using the definition in (3.3) we immediately obtain

$$
\sum_{p=0}^{k} \sum_{q=0}^{k} \lambda_{p} \bar{\lambda}_{q} R_{p, q}^{(N)}(\mathbf{x})=\lim _{n \rightarrow \infty} \frac{1}{2 n+1} \sum_{j=-n}^{n} \sum_{p=0}^{k} \sum_{q=0}^{k} \lambda_{p} \bar{\lambda}_{q} x_{j N+p} \bar{x}_{j N+q}
$$

Let ${ }^{T}$ denote the transpose of a vector and let $\underline{\mathbf{x}}:=\left(x_{j N}, \ldots, x_{j N+k}\right)$ and $\lambda=$ $\left(\lambda_{0}, \ldots, \lambda_{k}\right)$ be row vectors, we have for every $j$

$$
\sum_{p=0}^{k} \sum_{q=0}^{k} \lambda_{p} \bar{\lambda}_{q} x_{j N+p} \bar{x}_{j N+q}=\left|\lambda \underline{\mathbf{x}}^{T}\right|^{2} \geq 0,
$$

which gives the assertion.

And another important property is given in the following lemma. 
Lemma 3.8 If $\mathbf{x}=\left\{x_{j}\right\}_{j=-\infty}^{\infty}$ is a CS numerical sequence with period $N$, then

$$
\mathbf{y}=\mathbf{x}+\lambda \mathcal{S}^{p} \mathbf{x}, p \text { is an integer }
$$

is a CS numerical sequence with period $N$.

Proof: The claim about the mean is by assumption obvious. We need to first show that the autocorrelation kernel $R_{m, k}^{(N)}(\mathbf{y})$ for the sum exists. To verify this we consider the finite sum

$$
\begin{aligned}
\sum_{j=-n}^{n} y_{j N+m} \bar{y}_{j N+k}= & \sum_{j=-n}^{n}\left(x_{j N+m}+\lambda x_{j N+m+p}\right)\left(\overline{x_{j N+k}+\lambda x_{j N+k+p}}\right) \\
= & \sum_{j=-n}^{n} x_{j N+m} \bar{x}_{j N+k}+\bar{\lambda} \sum_{j=-n}^{n} x_{j N+m} \bar{x}_{j N+k+p}+ \\
& \lambda \sum_{j=-n}^{n} x_{j N+m+p} \bar{x}_{j N+k}+|\lambda|^{2} \sum_{j=-n}^{n} x_{j N+m+p} \bar{x}_{j N+k+p} .
\end{aligned}
$$

Dividing this equality by $2 n+1$ and taking the limit as $n \rightarrow \infty$, we obtain

$$
R_{m, k}^{(N)}(\mathbf{y})=R_{m, k}^{(N)}(\mathbf{x})+\bar{\lambda} R_{m, k+p}^{(N)}(\mathbf{x})+\lambda R_{m+p, k}^{(N)}(\mathbf{x})+|\lambda|^{2} R_{m+p, k+p}^{(N)}(\mathbf{x}) .
$$

Hence the required autocorrelation kernel $R_{m, k}^{(N)}(\mathbf{y})$ exists for every $m, k$ and

$$
R_{m, k}^{(N)}(\mathbf{y})=R_{m+N, k+N}^{(N)}(\mathbf{y}),
$$

showing that $\mathbf{y}$ is CS with period $N$.

It follows that any finite linear combination of shifts of a CS numerical sequence $\mathbf{x}$ is CS with the same period and therefore it is meaningful to consider the linear space $M_{N}(\mathbf{x})$ consisting of all such finite linear combinations:

$$
\mathbf{y}=\sum_{j=-p}^{p} \lambda_{j} \mathcal{S}^{j} \mathbf{x}, \text { arbitrary } p, \lambda_{j} .
$$

On $M_{N}(\mathbf{x})$ we may define a scalar product on two arbitrary elements $\mathbf{y}_{1}, \mathbf{y}_{2}$ by

$$
\left(\mathbf{y}_{1}, \mathbf{y}_{2}\right)_{N}=\sum_{j=1}^{n} \sum_{j^{\prime}=1}^{n^{\prime}} \lambda_{j}^{1} \overline{\lambda_{j^{\prime}}^{2}} R_{j, j^{\prime}}^{(N)}(\mathbf{x}) .
$$

Just as the scalar product (2.3) induces a norm on the elements of $M_{1}(\mathbf{x})$, the $N$-step scalar product (3.22) induces a norm on the elements of $M_{N}(\mathbf{x})$ provided we consider as identical any sequences $\mathbf{x}, \mathbf{y}$ with $\|\mathbf{x}-\mathbf{y}\|_{N}=0$. 
Proposition 3.9 A numerical sequence $\mathbf{x}$ is $C S(N)$ if and only if the numerical vector sequence

$$
\underline{\mathbf{x}}:=\left(\begin{array}{c}
\mathbf{x}^{(0)} \\
\mathbf{x}^{(1)} \\
\vdots \\
\mathbf{x}^{(N-1)}
\end{array}\right)
$$

formed in terms of $\mathbf{x}^{(k)}=\left\{x_{j N+k}\right\}_{j=-\infty}^{\infty} k=0,1, \ldots, N-1$ is jointly stationary in the sense of definition 2.2.

Proof: If $\mathbf{x}$ is CS with period $N$, then the existence of the limit $R_{m, n}^{(N)}(\mathbf{x})$ for arbitrary $m, n$ and the uniqueness of the relationships $m=p N+r$ and $n=q N+s$ shows that the limits $\phi_{r, s}(p-q)$ will exist for all $p, q \in \mathbf{Z}$ and $r, s \in[0,1, \ldots, N-1]$ and $\phi_{r, s}(p-q)=R_{m, n}^{(N)}(\mathbf{x})$. Further, the relationship $R_{m, n}^{(N)}(\mathbf{x})=R_{m+N, n+N}^{(N)}(\mathbf{x})$ makes it clear that $\phi_{r, s}(p-q)=R_{p N+r, q N+s}^{(N)}(\mathbf{x})$ depends only on the difference $p-q$.

The converse follows from the same relationships.

In view of the preceeding proposition, a totally stationary numerical sequence in the sense of definition 2.3 above is precisely a numerical sequence that is cyclostationary with every period.

The following does not occur in the case of stochastic sequences.

Proposition 3.10 If $\mathbf{x}$ is $C S(N)$ then it is $C S(1)$.

Proof: If $\mathbf{x}$ is $\operatorname{CS}(N)$ and the sum

$$
\frac{1}{2 M+1} \sum_{j=-M}^{M} \frac{1}{N} \sum_{k=0}^{N-1} x_{j N+m+k} \bar{x}_{j N+n+k}
$$

converges as $M \rightarrow \infty$, then the limit is $r_{m-n}(\mathbf{x})$. But the sum does indeed converge to

$$
\frac{1}{N} \sum_{k=0}^{N-1} R_{m+k, n+k}^{(N)}(\mathbf{x})=r_{m-n}(\mathbf{x})
$$

for every $m, n$, hence proving the claim.

Finally, we observe from the invariance of the time origin that

$$
\left\|\mathcal{S}^{j} \mathbf{x}-\mathcal{S}^{j} \mathbf{y}\right\|_{N}=0
$$

is valid for every $j$ if and only if it is valid for $j=0,1, \ldots, N-1$.

\subsection{The Wold Isomorphism}

We are now in position to extend the isomorphism to the cyclostationary case. 
A stochastic sequence $\xi=\left\{\xi_{j}\right\}_{j=-\infty}^{\infty}$ with $E\left[\left|\xi_{j}\right|^{2}\right]<\infty$ is called cyclostationary (CS), see [13], or periodically correlated with period $N$ (PC) if

$$
\mu_{n}=E\left[\xi_{n}\right]=\mu_{n+N}
$$

and if the autocorrelation satisfies

$$
R_{m, n}=E\left[\xi_{m} \overline{\xi_{n}}\right]=R_{m+N, n+N}
$$

for every $m$ and $n$. Since $\mu_{n}$ is periodic in $n$, it is equivalent to require the autocovariance to satisfy (3.27). If $\xi$ is CS with period $N=1$, then $\xi$ is (weakly) stationary, so for the proper CS-property we require $N>1$. It is well known that a CS random sequence with period $N$ can be obtained by interleaving the components of a stationary vector valued random sequence with $N$ components and vice versa (see [13] and [25]).

The Wold isomorphism for cyclostationary sequences follows in exactly the same manner as in the stationary case. One first establishes the correspondence on the two linear manifolds and then takes limits.

Proposition 3.11 Let $\mathbf{x}$ be a $C S(N)$ numerical sequence having autocorrelation $R_{m, n}^{(N)}(\mathbf{x})$ and let $J$ be the map from $M_{N}(\mathbf{x})$ into $\operatorname{sp}\left\{\xi_{k}, k \in \mathbf{Z}\right\} \subset H(\xi)$ defined for arbitrary complex $\lambda, \mu$ and integers $k, p$ by

$$
J\left(\lambda \mathcal{S}^{p} \mathbf{x}+\mu \mathcal{S}^{k} \mathbf{x}\right)=\lambda \xi_{p}+\mu \xi_{k}
$$

Then $J$ can be extended as an isometric Hilbert space isomorphism from a complex Hilbert space $H_{N}(\mathbf{x})$ containing $M_{N}$ to the complex Hilbert space $H(\xi)$, the closed linear span of a CS stochastic process $\xi$ that has autocorrelation function $R_{m, n}^{(N)}(\mathbf{x})$.

Proof: Since some $H_{N}(\mathbf{x})$ exists, at the very least via the process of abstract completion [26, p. 121, 124 - 125], the proposition holds through the continuity (boundedness) of $J$ on $M_{N}(\mathbf{x})$.

But again the results of Bass [1, loc. cit.] may be applied because the pre-Hilbert space $M_{N}(\mathbf{x})$ is a subspace of the Marcinkiewicz space $\mathcal{M}^{2}$ of sequences described in the previous section. To see this, suppose $\mathbf{z} \in M_{N}(\mathbf{x})$, then $(\mathbf{z}, \mathbf{z})_{N}=\|\mathbf{z}\|_{N}^{2}$ exists and is given by limit of the type (3.22). But if the limit exists for $\mathbf{z}$ certainly the limsup exists and so $\mathbf{z} \in \mathcal{M}^{2}$. The theorem of Bass implies that if $\mathbf{y} \in H_{N}(\mathbf{x})$ then $\mathbf{y} \in \mathcal{M}^{2}$ but also that if $\mathbf{y}$ and $\mathbf{z} \in H_{N}(\mathbf{x})$, then $(\mathbf{y}, \mathbf{z})_{N}$ exists as a limit.

And now we know there is a continuous invertible linear map

$$
J: H_{N}(\mathbf{x}) \rightarrow H(\xi)
$$

that preserves inner products and the respective topologies are those induced $(\cdot, \cdot)_{N}$ and $(\cdot, \cdot)_{L_{2}}$.

The following proposition, whose proof is simple and thus omitted, (see [17] for the continuous time case and [4] for a review), gives the connection between unitary operators and cyclostationary sequences. 
Proposition 3.12 A second order stochastic sequence $\xi=\left\{\xi_{j}\right\}_{j=-\infty}^{\infty}$ is periodically correlated or cyclostationary with period $N$ if and only if there exists a unitary operator $U$ on the Hilbert space $H(\xi)$ for which

$$
\xi_{j+N}=U \xi_{j}
$$

for every integer $j$.

From (3.28) we obtain

$$
J \mathcal{S}^{k} \mathbf{x}=U \xi_{k-N}=U J \mathcal{S}^{k-N} \mathbf{x}
$$

which produces

$$
U=J \mathcal{S}^{N} J^{-1}
$$

which means that $U$ and $\mathcal{S}^{N}$ are unitarily equivalent.

\subsection{Spectral Representations of CS Numerical Sequences}

Having established the Hilbert space isomorphism between $H_{N}(\mathbf{x})$ and the Hilbert space $H(\xi)$ of a $\mathrm{CS}(N)$ random sequence, we can use any of the representations for CS random sequences to produce a corresponding representation for CS numerical sequences.

Recall however from the discussion of (3.25) that in the case of cyclostationary numerical sequences of period $N,\left\|\Pi_{j}\{\mathbf{x}\}-\Pi_{j}\{\mathbf{y}\}\right\|_{N}=0$ is valid for every $j$ if and only if it is valid for $j=0,1, \ldots, N-1$.

We begin with observing that CS random sequences are strongly harmonizable [13] and therefore CS numerical sequences are also. This means that the spectral representation (2.21) still applies in the sense that

$$
\left\|\mathcal{S}^{k} \mathbf{x}-\sum_{m=1}^{n} \exp \left(i \lambda_{m} k\right)\left[Z\left(\lambda_{m}\right)-Z\left(\lambda_{m-1}\right)\right]\right\|_{N}^{2}
$$

converges to 0 as $n \rightarrow \infty$ (and norm of the partition $\left\{0=\lambda_{0}, \lambda_{1}, \ldots, \lambda_{n}=2 \pi\right\}$ tends to zero) except that the increments $\left[Z\left(\lambda_{m}-Z\left(\lambda_{m-1}\right)\right]\right.$ (which are sequences themselves) are no longer orthogonal but have the characteristic correlation structure belonging to CS (or periodically correlated) sequences [13]. The strong harmonizability implies that the correlation is given by

$$
R_{m, n}^{(N)}(\mathbf{x})=\int_{0}^{2 \pi} \int_{0}^{2 \pi} \exp \left(i m \lambda_{1}-i n \lambda_{2}\right) r_{Z}\left(d \lambda_{1}, d \lambda_{2}\right)
$$

where the measure $r_{Z}$ is of bounded variation on $[0,2 \pi] \times[0,2 \pi]$ which may be expressed as

$$
\int_{0}^{2 \pi} \int_{0}^{2 \pi}\left|r_{Z}\left(d \lambda_{1}, d \lambda_{2}\right)\right|<\infty
$$


A harmonizable sequence is CS if and only if the support of $r_{Z}$ is contained in the union $S=\bigcup_{k} S_{k}$ of straight lines

$$
S_{k}=\left\{\left(\lambda_{1}, \lambda_{2}\right) \in[0,2 \pi]^{2} \mid \lambda_{2}=\lambda_{1}-2 \pi k / N\right\} \text { for } k=-(N-1), \ldots(N-1) .
$$

This particular spectral representation retains the decomposition with respect to complex exponentials and the time invariance of the spectral measure; however, the spectral process $Z(\lambda)$ does not have orthogonal increments. A spectral process having orthogonal increments may be obtained but the spectral process will depend on time (see $[4,17])$.

\section{Concluding Remarks}

The isomorphism in [34] was evidently constructed in order to justify the optimal application on a unique time-series any linear operations that are, e.g., optimal for the ensemble of a stationary stochastic sequence in the sense of mean square. In this paper we have made some details of Wold's construction more precise and introduced the Wold isomorphism as an isomorphic mapping between cyclostationary numerical sequences and cyclostationary stochastic sequences in the sense of Hilbert spaces. The linear space of sequences generated by shifts of the unique sequence is the cornerstone needed in this.

In the present generalization of Wold's idea it holds that

$$
\text { totally stationary } \subset N \text {-stationary } \subset C S(N) \subset C S(1) \text {, }
$$

where the leftmost inclusion is an observation following the definition 2.3, the second inclusion from the right is Remark 3.1 and the rightmost inclusion is proposition 3.10. The rightmost inclusion in (4.34) is reverse to the similar relationship valid in the terminology of cyclostationary and stationary time-series introduced via the fractionof-time distributions. This is evidenced by the discussion and the Venn diagrams on [8, pp.24-25]. The inclusions in (4.34) are a by-product of the fact that different inner products are used to determine whether a sequence is $\operatorname{CS}(N)$ or $\operatorname{CS}(1)$. In the notation of [8, pp.24-25] it only makes sense to talk of stationary time-series as a class inside the class of cyclostationary time-series. This is matched in (4.34) by the inclusion of the $N$-stationary numerical sequences in $C S(N)$, in view of the remarks in (3.1) and in view of the definition of stationary times series in [8, p.23].

Wold noted that if $\left\{X_{n}\right\}$ is a stationary second order ergodic sequence, then almost all of the sample sequences will be isomorphic to $\left\{X_{n}\right\}$ in the sense described above. Similarly, suppose $\left\{X_{n}\right\}$ is a stochastic sequence defined on a probability space $(\Omega, \mathcal{F}, \mu)$ by $X_{n}(\omega)=f\left(S^{n} \omega\right)$ where $S$ is invertible and measurable but only $N$-stationary with respect to $\mu$, in the sense that $\mu\left(S^{-N} E\right)=\mu(E)$ for $E \in \mathcal{F}$. Then if the system $\left(\Omega, \mathcal{F}, \mu, S^{N}\right)$ is ergodic and $f\left(S^{n} \omega\right) \in L_{2}(\Omega, \mathcal{F}, \mu)$, the averages

$$
\frac{1}{2 M+1} \sum_{j=-M}^{M} X_{j N+m} \bar{X}_{j N+n}=\frac{1}{2 M+1} \sum_{j=-M}^{M} f\left(S^{j N+m} \omega\right) \overline{f\left(S^{j N+n} \omega\right)}
$$


converge for $(\mu)$-almost every $\omega$ to

$$
E X_{i N+m} \bar{X}_{i N+n}=\int_{\Omega} f\left(S^{n} \omega\right) \overline{f\left(S^{m} \omega\right)} d \mu .
$$

If $\mathcal{N}_{n, m}$ is the $\mu$-null set on which convergence to the indicated limit fails, then it is clear that the set $\Omega-\cup_{m, n} \mathcal{N}_{n, m}$ has full measure and on this set all the required limits exist. Each $\omega$ in this set produces a numerical sequence for which the averages of (3.3) converge, and each such sequence is isomophic in the sense of Wold to the stochastic sequence.

For other issues related to ergodic theory of CS sequences, see Gray and Kieffer [14], Boyles and Gardner [2], and Gray [15].

Acknowledgments: Part of the research reported here was done during a visit by the first author at Luleå University of Technology. The support by the School of Engineering and the Division of Signal Processing of the Luleå University of Technology as well as the generous hospitality of prof. Per Ola Börjesson are hereby gratefully acknowledged.

This work was supported in part by the Office of Naval Research under contracts N00014-92-C-0057 and N00014-95-C-0093, and by the U.S. Army Research Office under contract DAAH04-96-C-0027.

\section{References}

[1] J. Bass, Fonctions de Corr'elation Fonctions Pseudo-Al'eatoires et Applications, Masson, New York, 1984.

[2] R. A. Boyles and W. A. Gardner, Cycloergodic Properties of Discrete-Parameter Nonstationary Stochastic Processes, IEEE Trans. on Information Theory, vol. IT-29, no. 1, pp.105-114, 1983.

[3] D. R. Brillinger, Time Series, Data Analysis and Theory, Holden Day, San Francisco, 1981.

[4] D. Dehay and H. Hurd, Representation and Estimation for Periodically and Almost Periodically Correlated Random Processes, in Cyclostationarity in Communications and Signal Processing, W.A. Gardner, ed., IEEE Press, 1993, pp. 295-328.

[5] H. Furstenberg, Stationary Processes and Prediction Theory, Annals of Mathematics Study, No. 44, Princeton University Press, Princeton, N. J. 1960.

[6] W. A. Gardner, Introduction to Random Processes With Applications to Signals \& Systems, 2nd Edition, McGraw-Hill, New York. 1990. 
[7] W. A. Gardner, Statistical Spectral Analysis, A Nonprobabilistic Theory, Prentice Hall, Englewood Cliffs, NJ, 1987.

[8] W. A. Gardner, An Introduction to Cyclostationary Signals, in Cyclostationarity in Communications and Signal Processing, W.A. Gardner, ed., IEEE Press, 1993, pp. 1-90.

[9] W. A. Gardner and W. A. Brown, Fraction-of-time probability for time-series that exhibit cyclostationarity, Signal Processing, 23, 1991, pp. 273 - 292.

[10] W. A. Gardner, Two Alternative Philosophies for Estimation of the Parameters of Time-Series, IEEE Trans. on Information Theory, vol. 37, no. 1, pp. 216-218, 1991.

[11] W. A. Gardner and C. M. Spooner, The cumulant theory of cyclostationary time series, Part I: Foundation, IEEE Trans. Signal Processing, vol. 42, pp. 3387-3408, December 1994.

[12] C. M. Spooner and W. A. Gardner, The cumulant theory of cyclostationary time series, Part II: Development and applications, IEEE Trans. Signal Processing, vol. 42, pp. 3409-3429, December 1994.

[13] E. G. Gladyšev, Periodically Correlated Random Sequences, Sov. Math., 2, 1961, pp. $338-388$.

[14] R. M. Gray and J. C. Kieffer, Asymptotically mean stationary measures, Ann. Prob., 8, 1980, pp. 962 - 973.

[15] R. M. Gray, Probability, Random Processes and Ergodic Properties, SpringerVerlag, New York, 1988.

[16] H. L. Hurd, An Investigation of Periodically Correlated Stochastic Processes, Ph. D. -thesis, Dept. of EE, Duke University, 1969.

[17] H. L. Hurd and G. K. Kallianpur, Periodically Correlated Processes and Their Relationship to $L_{2}[0, T]$-Valued Stationary Sequences, in Nonstationary Stochastic Processes and their Application, A.G. Miamee, Ed., World Scientific Publishing Co., pp. 256-284, 1992.

[18] H. L. Hurd and T. Koski, Spectral Theory of Cyclostationary Arrays, submitted to the volume in honor of M.M. Rao.

[19] L. Izzo and A. Napolitano, Higher-order cyclostationary properties of sampled time-series, Signal Processing, 54, 1996, pp. 303-307.

[20] L. Izzo and A. Napolitano, Higher-order statistics for Rice's representation of cyclostationary signals, Signal Processing, 56, 1997, pp. 279-292. 
[21] L. Ljung, System Identification. Theory for the User, Prentice-Hall Inc. Englewood Cliffs, N. J. 1987.

[22] G. W. Mackey, Ergodic Theory and its Significance for Statistical Mechanics and Probability Theory, Adv. in Math., 12, 1974, pp. 178 - 268.

[23] P. Masani, Review of [5], Bull. AMS, 69, March 1963, pp. 195 - 207.

[24] P. Masani, Einstein's Contribution to Generalized Harmonic Analysis, Jahrbuch Überblicke Mathematik, 1986, pp. 191- 209.

[25] A.G. Miamee and H. Salehi, On the Prediction of Periodically Correlated Stochastic Processes, in P. R. Krishnaiah (ed.), Multivariate Analysis V, 1980, pp. 167 - 179 .

[26] A. W. Naylor and G. R. Sell, Linear Operator Theory in Engineering and Science, Springer-Verlag, New York, Heidelberg, Berlin, 1982.

[27] Yu. A. Rozanov, Stationary Random Processes, Holden-Day, San Francisco, Cambridge, London, Amsterdam, 1967.

[28] M. H. Stone, Linear Transformations in Hilbert Space, American Mathematical Society Colloquium Publications, Vol. 15, Providence R. I. 1932.

[29] N. Wiener, The spectrum of an array, J. of Math. Phys., 6, 1927, pp. 145-157.

[30] N. Wiener, Generalized Harmonic Analysis, Acta Math., 55, 1930, pp. 117 - 258.

[31] J. C. Willems, From Time Series to Linear System- Part I. Finite Dimensional Linear Time Invariant Systems, Automatica, 22, 1986, pp. 561 - 580.

[32] J. C. Willems, From Time Series to Linear System- Part II. Exact Modelling, Automatica, 22, 1986, pp. 675 - 694 .

[33] J. C. Willems, From Time Series to Linear System- Part III. Approximate Modelling, Automatica, 23, 1987, pp. 87 - 115.

[34] H. O. A. Wold, On Prediction in Stationary Time Series, Ann. Math. Stat. , 19, 1948, pp. 558 - 567. 